\title{
Repeating microlensing events in the OGLE data
}

\author{
Jan Skowron ${ }^{* 1}$, Łukasz Wyrzykowski ${ }^{1,2}$, Shude Mao ${ }^{3}$ \\ ${ }^{1}$ Warsaw University Astronomical Observatory \\ Al. Ujazdowskie 4 \\ 00-478 Warszawa, Poland \\ E-mail: jskowroneastrouw.edu.pl \\ ${ }^{2}$ Institute of Astronomy, University of Cambridge \\ Madingley Road \\ CB3 OHA Cambridge, UK \\ E-mail: wyrzykow@ast.cam.ac.uk \\ 3 Jodrell Bank Centre for Astrophysics \\ Alan Turing Building, The University of Manchester \\ M13 9PL Manchester, UK \\ E-mail: shude.mao@manchester.ac.uk
}

\begin{abstract}
Microlensing events were usually selected from single-peaked, non-repeating light curves in order to avoid confusions with variable stars. However, it is possible that the "same" object experiences second microlensing brightening episode. For example, two separate events may occur because either the source or the lens is a binary system. Previous theoretical studies predict a small fraction, up to few precent, of events should exhibit repeating microlensing phenomenon. A careful analyses of such events provides an important way to study the wide binary population and detect extrasolar planets. The number and importance of these events will increase considerably when the next-generation wide-field microlensing experiments come online.

We searched light curves of about 4000 microlensing events detected by the Optical Gravitational Lensing Experiment (OGLE) in the Galactic Bulge from 1992 to 2007, including those discovered by the Early Warning System (EWS) and other independent studies. Our search revealed a total of 19 repeating candidates, with 6 clearly due to wide binary lens. We also found that a total of 64 events $(\sim 3.6 \%$ of the total) were mis-classified as microlensing; mostly of these were nova-like or other eruptive stars, including 24 dwarf novae.
\end{abstract}

The Manchester Microlensing Conference: The 12th International Conference and ANGLES Microlensing Workshop

January 21-25 2008

Manchester, $U K$

*Speaker. 


\section{Introduction}

Standard microlensing events are assumed to single-peaked and is constant in the baseline before and after the event. [1] studied the possibility of repeating microlensing due to wide binary lenses and predicted that $0.5-2 \%$ of observed microlensing curves should exhibit apparent repetition. To date only one such event was found and studied in detail [3]. Apart from the wide binary lenses there are also other microlensing scenarios that may lead to the similar features in the light curves. For example, when the source itself is a wide binary, the light of the second star can be significantly magnified after the first source has already returned to the baseline. Also one lens can magnify two unrelated stars blended in one seeing disk (about 1 arc second). Finally two unrelated microlensing events (due to two lenses) can occur for two unresolved stars within the seeing disk. All these scenarios can produce two separated magnification bumps in the light curve, although the latest two have a much lower probability of occurrence. In this work we focus only on the wide binary source and wide binary lens scenarios.

\section{Observational data}

In this study we used data acquired by the Optical Gravitational Lensing Experiment (OGLE) [7]. We gathered all the available data on events detected by the OGLE's Early Warning System (EWS) ${ }^{1}$ from years 1998-2000 (OGLE-II) and 2001-2007 (OGLE-III) [7]. On top of that we also added events found independently in the OGLE-II 1997-1999 data by [8] and in the OGLE-III 2001-2005 data by [9] and [10]. In total there are 4135 events, with 154 duplicate entries.

\section{Search procedures}

Each event was checked if there is any additional observational data available in the OGLE-I (1992-1995), OGLE-II and OGLE-III databases. If the star was observed during all three phases of the OGLE project its light curve spanned for up to 15 years (1992-2007). There were about 2300 objects with 5 years of continuous observations, about 1200 observed for 10 years and 152 objects were monitored for about 15 years.

The resulting light curves were investigated for the presence of two or more magnification episodes in the whole spanning time. All light curves were analysed in two ways: by visual inspection and by an automated algorithm; we discuss these in turn.

\subsection{Visual inspection}

Systematic visual inspection of all 4135 light curves revealed a sample of events which were misclassified as microlensing earlier. Contamination was mainly by nova-like outbursts and other variables, mostly eruptive stars. Availability of long time span of the light curves provided an opportunity for better classification of the events, e.g. identifying another outbursts or another period in oscillations.

For the largest and the most uniform sample of 3159 microlensing candidates from the EWS from OGLE-III phase only (until September 2007), $64(\sim 2 \%)$ turned out to be variable stars,

\footnotetext{
${ }^{1}$ http://www.astrouw.edu.pl/ ogle/ogle3/ews/ews.html
} 
among which 24 showed a behavior similar to dwarf novae stars (multiple, short-time outbursts). 52 events $(\sim 1.6 \%)$ had duplicate entries due to overlaps of adjacent OGLE fields.

From the visual search a total of 13 candidates for repeating events were found.

\subsection{Automated search algorithm}

The main goal for developing an automated algorithm was to find repeating events in which the second magnification episode is considerably smaller than the first one, as such configuration is hard to find by eye. Because of the contamination of our event sample with variable stars and caustic crossing events, we constructed a semi-automatic algorithm, which still requires some human supervision, but ensure that none of visually found candidates were lost.

In the first step we detect the main microlensing episode and fit it with the Paczyński's light curve [巨]]. Then the main bump was cut out from the light curve. A constant light curve model and the Paczyński model were then fit to the remaining data, yielding two $\chi^{2}$ s: $\chi_{2 \text { nd,const }}^{2}, \chi_{2 \mathrm{nd}, \mathrm{Pac}}^{2}$. If there was a repeating event present, $\chi_{2 \text { nd,Pac }}^{2}$ should be substantially smaller than $\chi_{2 \text { nd,const }}^{2}$.

To ensure the second fit is reliable, we have calculated the number of data points enclosed inside the second magnification episode, $n_{2 \text { nd }}$, and rejected all events with $n_{2 \text { nd }}<3$ or $n_{2 \text { nd }}>N / 2$, where $N$ is the total number of data points. We construct the following statistics:

$$
s=\frac{\left|\chi_{2 \mathrm{nd}, \mathrm{Pac}}^{2}-\chi_{2 \mathrm{nd}, \mathrm{const}}^{2}\right|}{\chi_{2 \mathrm{nd}, \mathrm{Pac}}^{2}},
$$

and choose events with $s<0.2$.

A total of 193 events passed the described criteria. All of them were then carefully examined visually and we retrieved all the 13 events from the visual search (see §3.1) and uncovered 6 new candidates, bringing the total number of candidates for repeating events to 19 .

3 out of 19 of our candidates were previously found in other studies: the correlation between two EWS events OGLE-1999-BUL-42 and OGLE-2003-BLG-220 was found by [П†; OGLE174828.55221639.9 was found by [9] and OGLE-2003-BLG-291 was described in [⿰]].

\section{Modelling}

All 19 events were fitted with three different models: binary source, binary lens and approximate wide binary lens.

We model a binary lens following [6] with point-like masses described by the mass ratio $(q)$ and separation $(d)$ in units of Einstein radius $\left(r_{E}\right)$. Microlensing parameters in these models are: impact parameter $(b)$, angle between source trajectory and projected binary axis $(\beta)$, Einstein radius crossing time $\left(t_{E}\right)$ and the time of minimal approach to the binary mass center $\left(t_{0}\right)$. Two flux parameters are included: magnitude of the baseline of the event $\left(I_{0}\right)$ and fraction of the light contributed by the lensed source to the blend ( $f ; f=1$ indicates no blending). In the fitting we used point source approximation. In some cases in order to better fit the observational data we introduced the Earth parallax motion (with a parallax scale $\pi_{E}$ ) or rotation of the binary lens (with an angular velocity of $\dot{\beta}$ ).

The search for the best models was conducted on a grid covering a wide range for all the parameters with fluxes of the baseline and the source calculated analytically. 


\begin{tabular}{|c|c|c|c|c|}
\hline Event OGLE- & $\left(f_{1} / f_{2}\right)_{b s}$ & $q_{b l}$ & $q_{\text {approx }}$ & Type \\
\hline 1999-BUL-42 & \multicolumn{3}{|c|}{ model not found } & $\mathrm{cc} \mathrm{bl}$ ? \\
\hline 1999-BUL-45 & 0.189 & 0.203 & 0.270 & $\mathrm{bl} / \mathrm{bs}$ \\
\hline 2000-BUL-42 & 0.260 & 0.339 & 0.400 & $\mathrm{bl}$ \\
\hline 2002-BLG-018 & 0.280 & 0.395 & 0.443 & $\mathrm{bs} / \mathrm{bl}$ \\
\hline 2002-BLG-045 & 0.045 & 0.008 & 0.011 & bl \\
\hline 2002-BLG-128 & - & 0.611 & - & $\mathrm{cc} \mathrm{bl}$ \\
\hline 2003-BLG-063 & 0.539 & 0.203 & 0.428 & $\mathrm{bl}$ \\
\hline 2003-BLG-067 & 0.817 & 0.788 & 0.844 & $\mathrm{bs} / \mathrm{bl}$ \\
\hline 2003-BLG-126 & 0.292 & 0.604 & 0.918 & $\mathrm{bl}$ \\
\hline 2003-BLG-291 & - & 0.617 & - & $\mathrm{cc} \mathrm{bl}$ \\
\hline 2003-BLG-297 & 0.075 & 0.147 & 0.121 & $\mathrm{bl} / \mathrm{bs}$ \\
\hline 2004-BLG-075 & 0.707 & 0.587 & 0.664 & $\mathrm{bl} / \mathrm{bs}$ \\
\hline 2004-BLG-328 & 0.150 & 0.056 & 0.054 & bs \\
\hline 2004-BLG-440 & 0.927 & 0.622 & 0.906 & $\mathrm{bl}$ \\
\hline 2004-BLG-591 & 0.548 & 0.507 & 0.431 & bl \\
\hline 2006-BLG-038 & - & 0.569 & - & $\mathrm{cc} \mathrm{bl}$ \\
\hline 2006-BLG-460 & 0.388 & 0.267 & 0.285 & $\mathrm{bl}$ \\
\hline $175257.97-300626.3$ & - & 0.195 & - & $\mathrm{cc} \mathrm{bl}$ \\
\hline $174828.55-221639.9$ & _- & 0.158 & _- & $\mathrm{cc} \mathrm{bl}$ \\
\hline
\end{tabular}

Table 1: Candidates for repeating microlensing events. Mass ratios obtained in the standard binary model $\left(q_{\mathrm{bl}}\right)$ and approximate wide binary model $\left(q_{\text {approx }}\right.$, see $\left.\S 4\right)$ are shown, as well as the light ratio of sources in the binary source model. "bl" indicates that binary lens model is considerably better than the binary source model; "bs" means that binary source model is better; "bl/bs" indicates that both models have comparable $\chi^{2}$,s. For events with clear caustic crossing features ("cc bl") only the standard binary lens model was fitted.

We used simple static binary source model: the predicted light curve is a sum of two standard single microlensing events with two impact parameters $\left(b_{1}\right.$ and $\left.b_{2}\right)$, two times of the maximum magnification ( $t_{01}$ and $\left.t_{02}\right)$, separate flux fractions of each source $\left(f_{1}, f_{2}\right)$, baseline magnitude $\left(I_{0}\right)$ and Einstein radius crossing time $\left(t_{E}\right)$. To ensure the resulting models are comparable with the binary lens models we used a similar minimisation strategy with the same grid sizes and the same optimisation routines as that for the binary lens model.

In addition, we use an approximate wide binary model following the concept of [1]. They noted that for a repeating event it is possible to obtain the mass ratio of the binary lens simply from the ratio of the squares of the time scales of two magnification peaks. To test this method we have constructed a simplified model in which the binary lens acts as the sum of two independent point-like lenses. Each lens has a magnification $\mu_{i}(i=1,2)$ and the resulting magnification was $\mu \approx \mu_{1}+\mu_{2}-1$. Fitting was done on a grid similar to that for the rigourous binary lens model.

\section{Results}

Table 1 lists all 19 candidates for repeating events and presents mass ratios in the standard binary model and approximate wide binary model and the light ratio obtained in the binary source model. The last column indicates the type of event as concluded from the best value of $\chi^{2}$ of the binary lens and binary source fits. These models are regarded as comparable when their $\chi^{2}$, differ less than 10. "bl" stands for binary lens and "bs" for binary source. "cc bl" indicates that the caustic crossing features were clearly visible in the light curve, therefore only the "bl" model was studied. 
Light curves of all 19 events are presented in the Appendix with the best full binary lens model shown, where possible.

For OGLE-1999-BUL-42/OGLE-2003-BLG-220 we were not able to find any satisfactory model. In 1999, the event showed some features resembling a caustic crossing, which in principle can also be explained with a binary source model. However, simple static binary source model was not sufficient. The other peak, which occurred about 4 years later, shows nearly no binarity features but only one outlying data point and a slight asymmetry. It requires further detailed studies in order to reject or confirm the hypothesis that these were in fact two independent sources magnified by one binary lens or just two completely independent events occurring in one seeing disk.

For OGLE-2002-BLG-045 the second brightening was much shorter than the first one and the binary lens models gave an extreme mass ratio of $q=0.008$. Unfortunately, sparse samplings of both peaks prevent us from concluding convicingly the planetary nature of the lens. Nevertheless this demonstrates a potential channel of detecting planets in events which has already finished their single event phase (e.g., [2]).

Fitting results for event OGLE-2003-BLG-291 were taken from [3].

Clear caustic crossing features were present in the light curves of about $30 \%$ of our candidates, which is of an order of magnitude more than in the sample of all microlensing events- in the uniform sample of 3159 candidates from EWS in OGLE-III there were 73 events with clear caustic crossing which gives only $\sim 2.4 \%$.

To answer the question how long observers should wait for the second brightening to occur, we calculated the time between the peaks and the results are presented in Figure 1 (left panel) vs. the event time scales. As predicted in [1] this time is of order of a just a few Einstein times; for the events here, between 32 to 472 days, with a median of 142 days.

The right panel of Figure 11 presents the comparison of the mass ratios in the full and approximate binary lens models. A strong correlation is clearly visible, indicating that the simple approximate model can be used for an estimation of the mass ratio in the wide binary lenses with separate magnification peaks.

\section{Conclusions}

Microlensing events do repeat. We found 19 repeating candidates, about $\sim 0.5 \%$ of the sample of 4135 microlensing events studied here. This gives an occurrence rate of $\sim 2 /$ year. Both the overrepresentation of caustic crossing events and comparison of the goodness of fit between binary lens and binary source fits suggest that probably most of these are due to wide binary lenses.

We showed that it is possible to estimate the mass ratios for repeating events in a simple way. With a growing number of microlensing events observed every year these events could be a straightforward and independent way for studying the wide stellar binary populations in the Galaxy.

We also showed an example of "missed opportunity" for finding a planet, as the sampling was not dense enough after the event. In the future it would be profitable for survey teams and followup networks to pay more attention to microlensing events even well after the main magnification peak. The number of repeating events will increase considerably when the next-generation widefield microlensing surveys come online. The dense sampling will be particularly important for the detection of extrasolar planets on wide orbits and will offer a new channel of extrasolar planet 

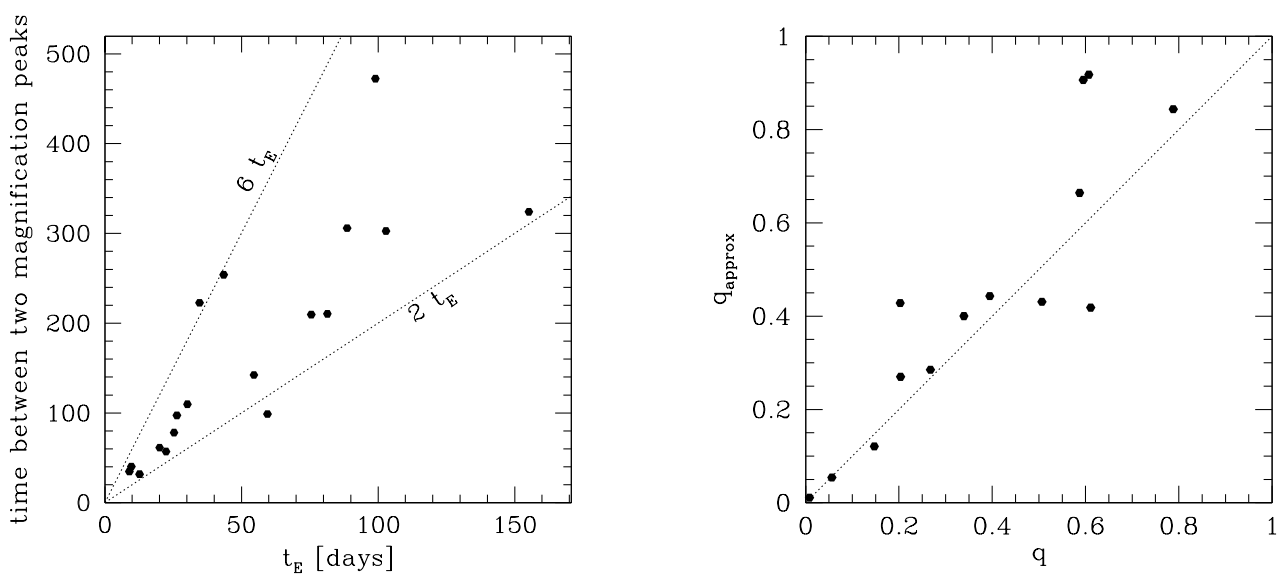

Figure 1: Time between two peaks in repeating events versus the Einstein radius crossing time, $t_{E}$ (left panel). The right panel shows the correlation between mass ratios obtained in the full $(q)$ and approximate $\left(q_{\text {approx }}\right)$ binary lens models (see $\left.\S 4\right)$.

discovery [䜣. As we demonstrated in this work, the mass ratios in such cases can be "read" off from the light curves from the approximate binary lens model (see Fig. 1, right panel).

As a by-product of our search we found that the contamination with non-microlensing events in the OGLE-III Early Warning System is 3.6\% (64 misclassified events). The main contributors are the dwarf novae (24).

\section{Acknowledgments}

We thank Drs. Andrzej Udalski, Michał Jaroszyński and Martin Smith for their support and discussions. The authors acknowledge support of the European Community's FR6 Marie Curie Research Training Network Programme, Contract No. MRTN-CT-2004-505183 "ANGLES". This work was supported in part by Polish MNiSW grants N203 008 32/0709 and N203 030 32/4275.

\section{References}

[1] Di Stefano, R., Mao, S. 1996, ApJ, 457, 93

[2] Di Stefano, R., Scalzo, R. A. 1999, ApJ, 512, 579

[3] Jaroszyński, M., et al. 2005, Acta Astronomica, 55, 159

[4] Klimentowski J., 2005, Masters Thesis, Warsaw University

[5] Paczyński, B. 1986, ApJ, 304, 1

[6] Skowron, J., et al. 2007, Acta Astronomica, 57, 281

[7] Udalski, A. 2003, Acta Astronomica, 53, 291

[8] Woźniak, P. R., et al. 2001, Acta Astronomica, 51, 175

[9] Wyrzykowski Ł., 2005, PhD Thesis, Warsaw University Astronomical Observatory

[10] Wyrzykowski, Ł., et al. 2006, Acta Astronomica, 56, 145 


\section{A. Appendix. Light curves of 19 repeating microlensing events together with the} best-fit binary lens model.
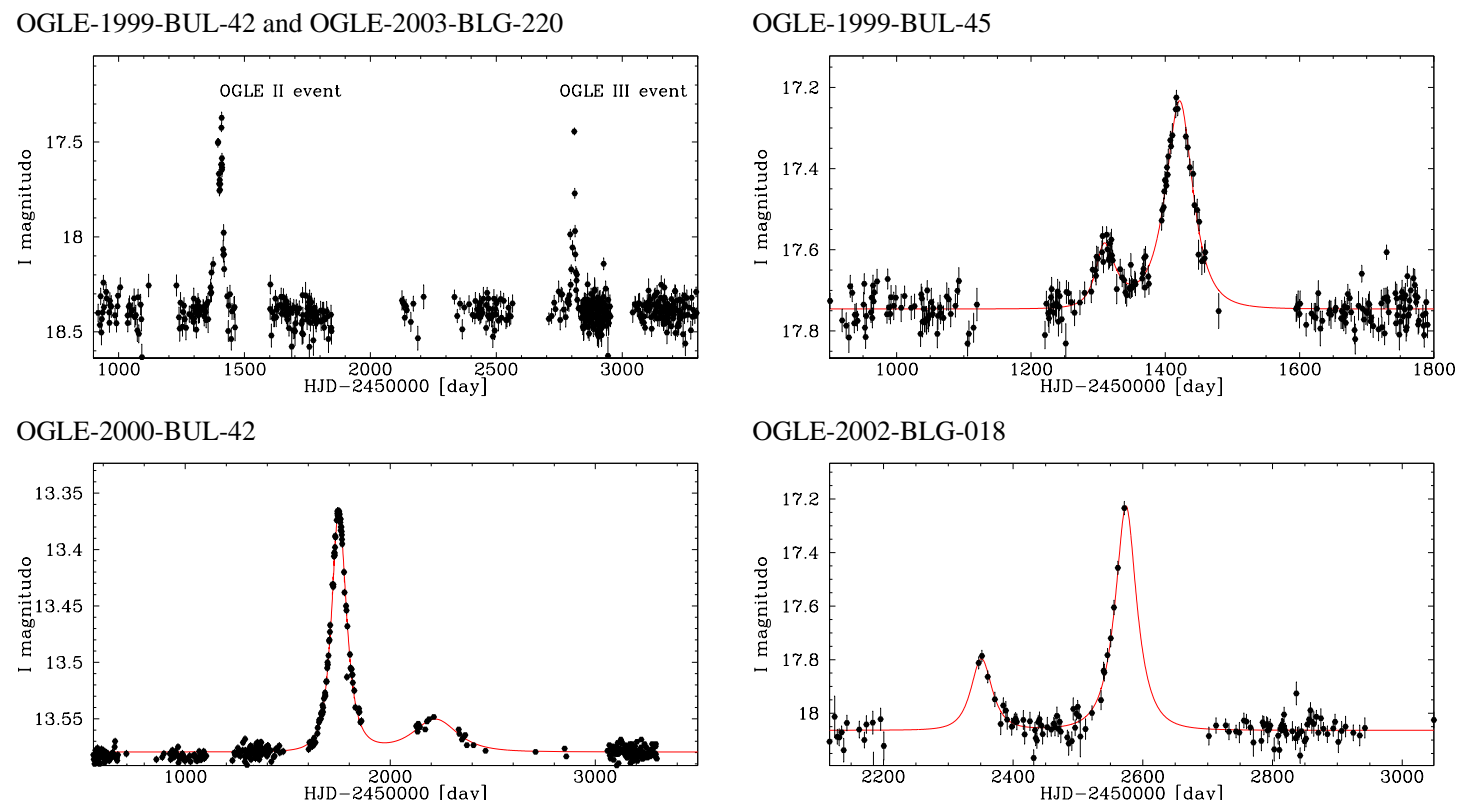

OGLE-2002-BLG-018

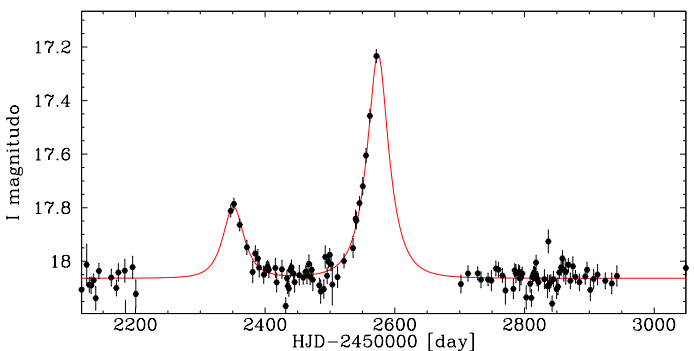

OGLE-2002-BLG-045
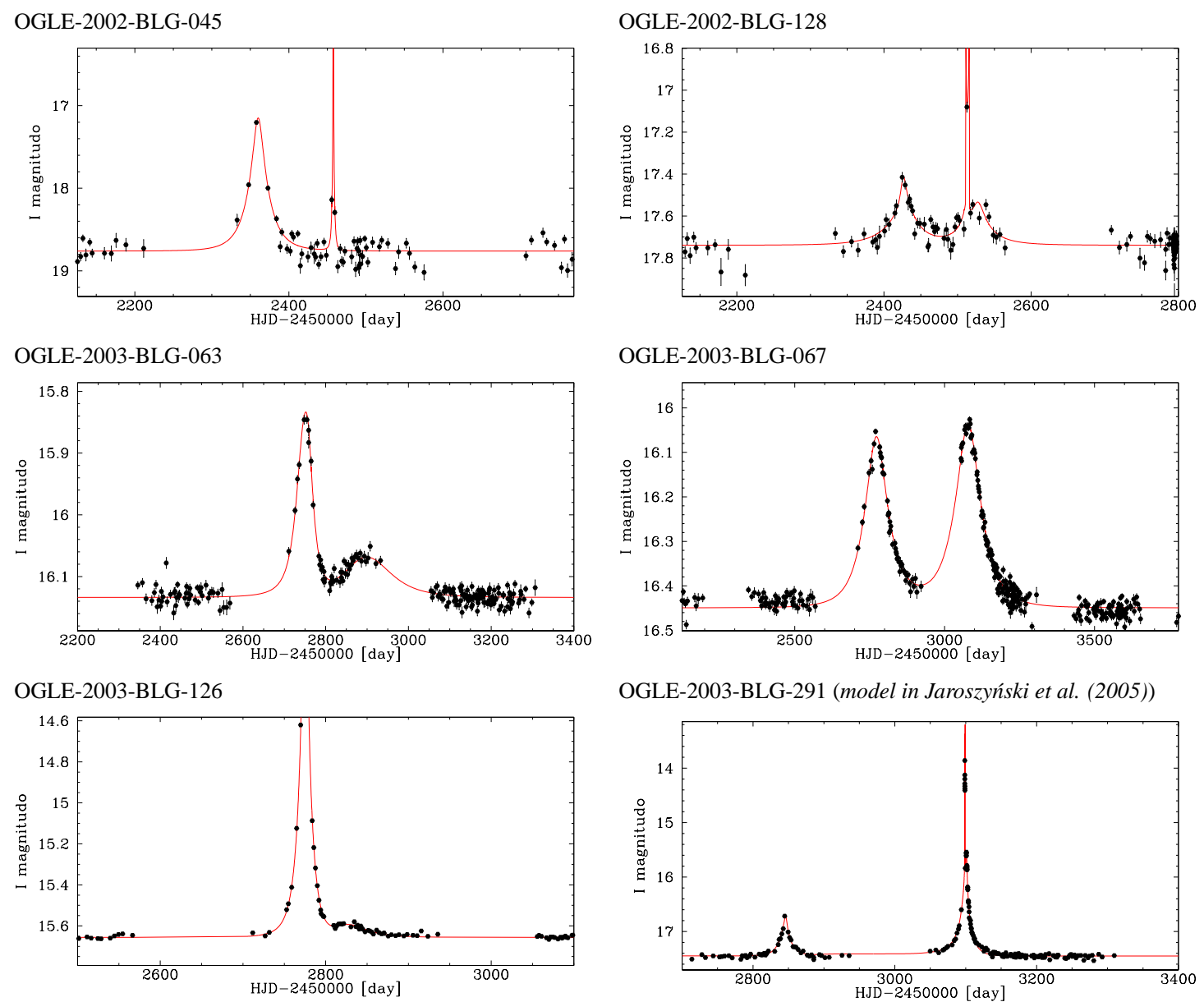

OGLE-2003-BLG-291 (model in Jaroszyński et al. (2005))

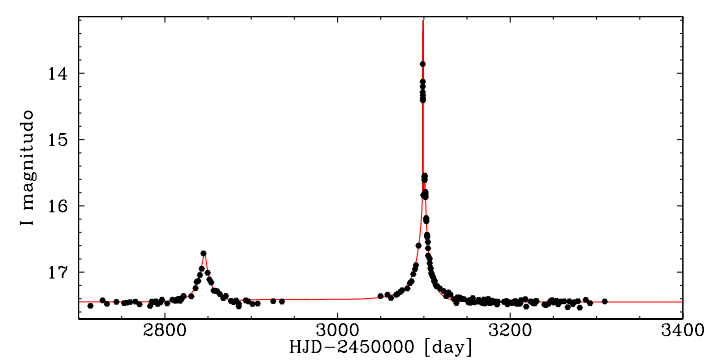



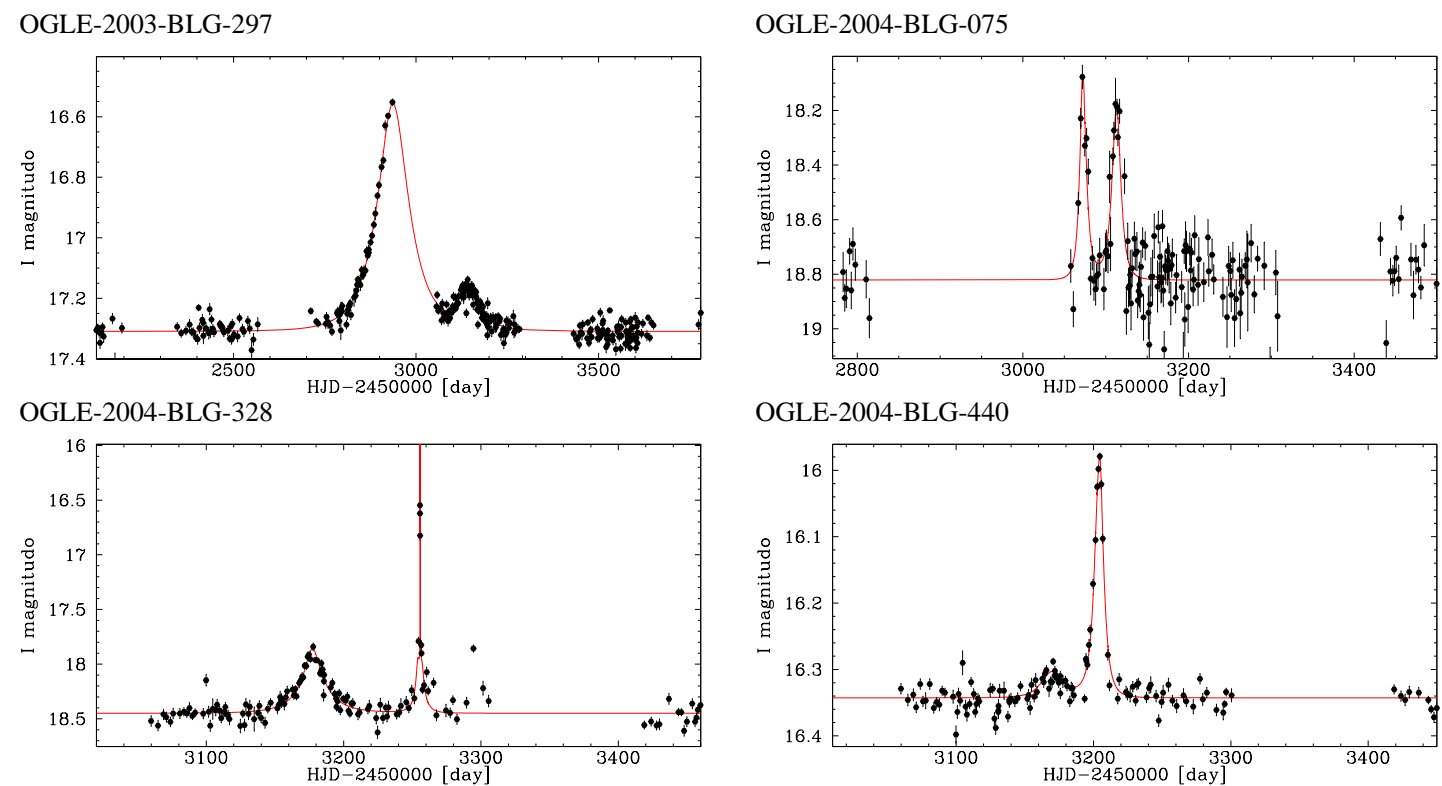

OGLE-2004-BLG-440

OGLE-2004-BLG-591
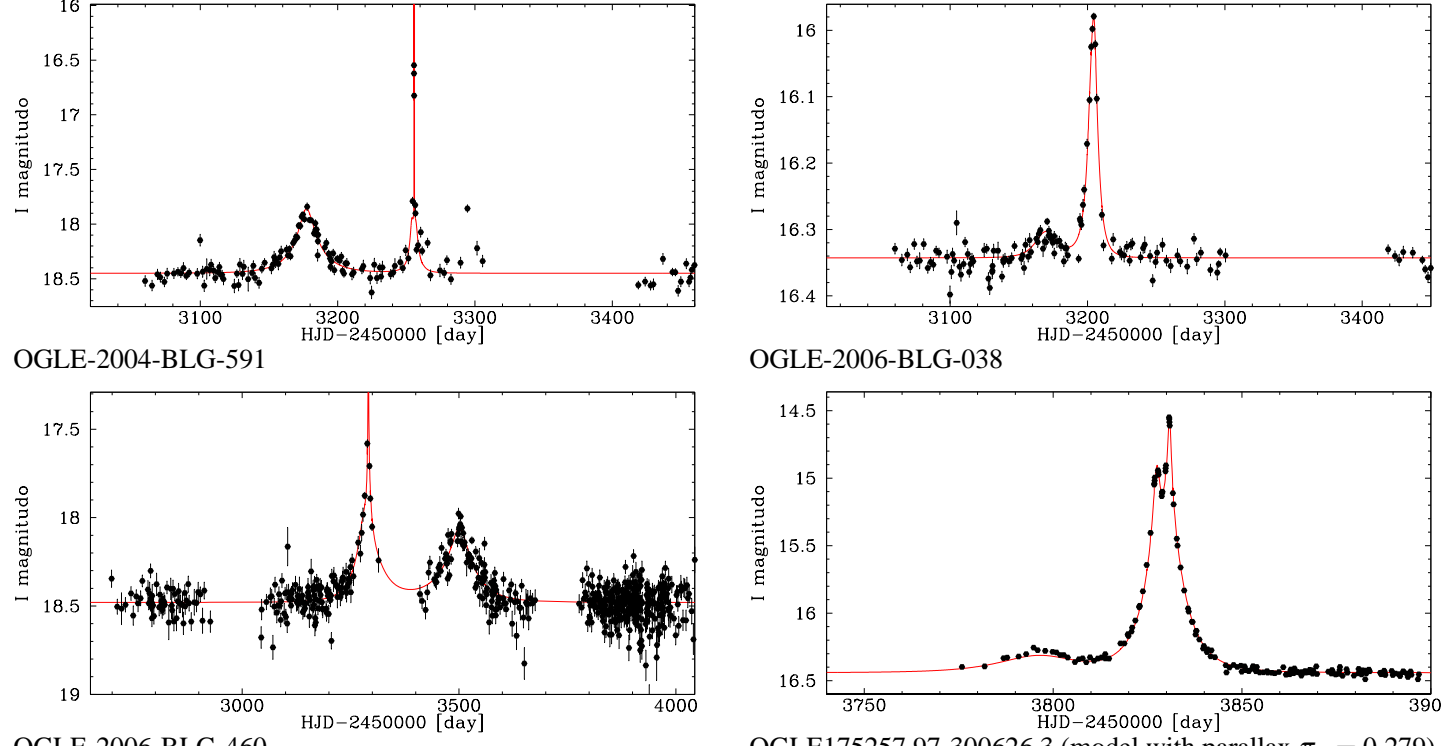

OGLE-2006-BLG-038
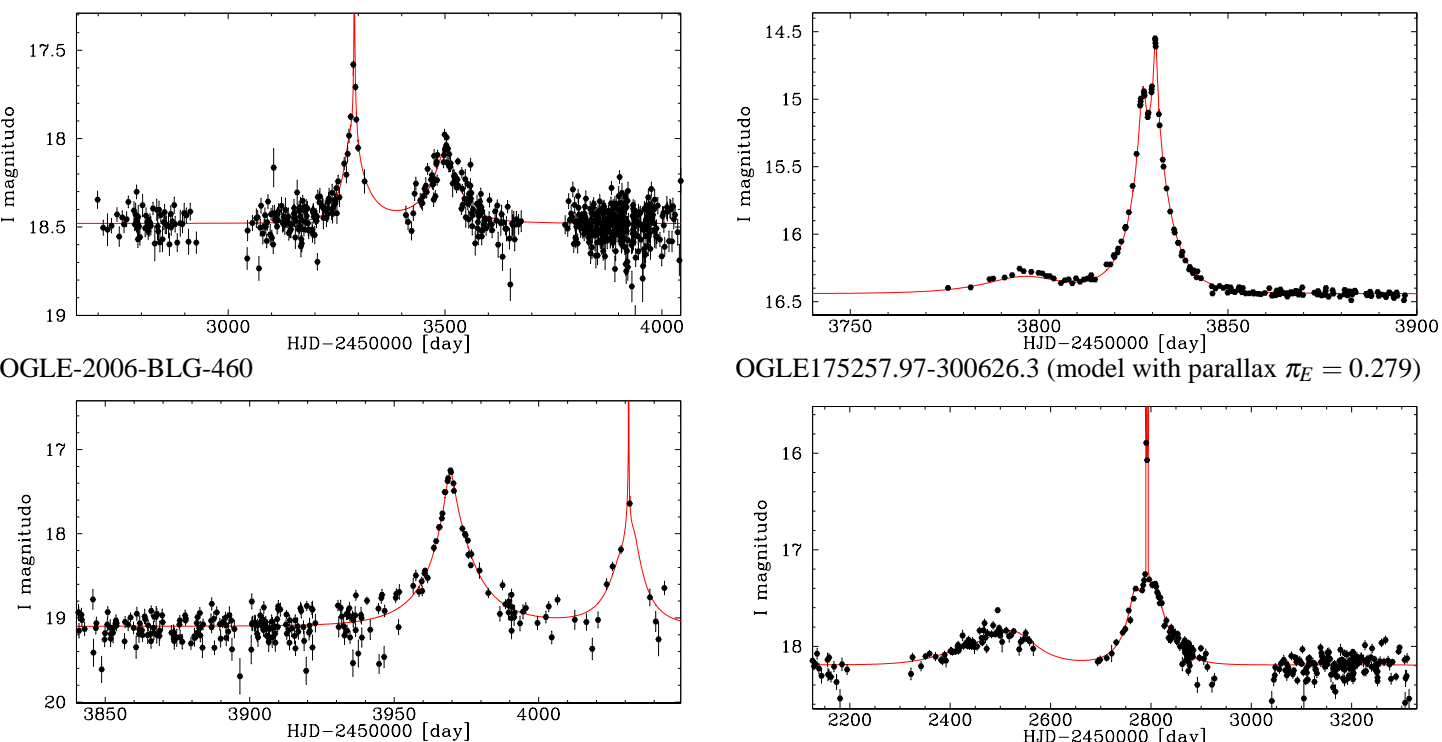

OGLE175257.97-300626.3 (model with parallax $\pi_{E}=0.279$ )

OGLE174828.55-221639.9 (model with axis rotation $\dot{\beta}=$ $0.032^{\circ} /$ day)
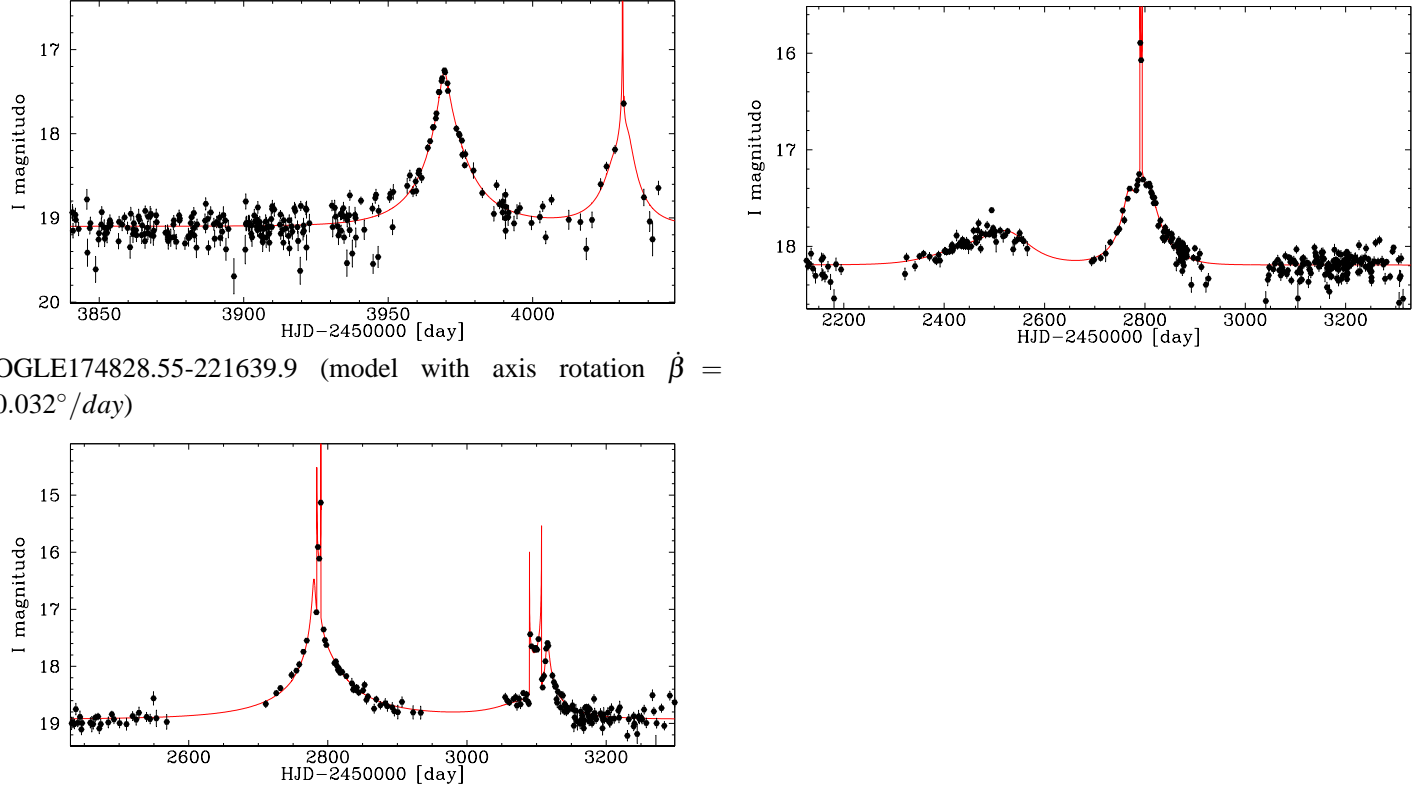